Als erstes war das immer wieder ins Feld geführte Argument zu entkräften, die Richterinnen wollten nicht an die obersten Bundesgerichte und es gäbe daher zu wenige geeignete Frauen. Angesichts 8.000 Richterinnen in Deutschland und den wenigen Vakanzen an den obersten Bundesgerichten pro Jahr (bei dieser Richterwahl gerade mal 17 Bundesrichter/innen/stellen) ist dieses Argument seit Langem widerlegt. Es fehlt nicht am Angebot, sondern an der Nachfrage und an einer systematischen Personalentwicklung. Artikel 3 Absatz 2 GG war und ist keine Kann-Vorschrift, sondern Verpflichtung und Auftrag für alle Vorgesetzten.

Mit Hilfe des djb-Netzwerks und von Gleichstellungsbeauftragten habe ich daher hoch qualifizierte Richterinnen gesucht und, wie erwartet, auch gefunden. Allein sechs der 13 Kandidatinnen für den BGH sind mit Hilfe des djb auf die Vorschlagslisten gekommen. Bei der Suche waren djb-Kolleginnen von den Oberlandesgerichten und Oberverwaltungsgerichten bzw. Verwaltungsgerichtshöfen behilflich. Richterinnen an den obersten Bundesgerichten nannten die Namen von wissenschaftlichen Mitarbeiterinnen. Einige Kolleginnen fanden den Mut, sich direkt an mich zu wenden. Auch die Kontakte zu den Leitungen der Bundesgerichte waren fruchtbar. Dort wird die Notwendigkeit, die Senate mit mehr Richterinnen zu besetzen, weitgehend anerkannt.

Auf meiner Liste stehen zwar bereits die Namen einiger Kolleginnen, die aufgrund hervorragender Qualifikation bei den nächsten Wahlen punkten können. Ich benötige aber für die Richterwahl 2013 weitere Vorschläge, insbesondere auch für BAG und BSG. Bitte nennen Sie mir geeignete Kolleginnen bzw. wenden Sie sich unmittelbar an mich. Dass ich dies vertraulich behandle, ist selbstverständlich.

Da die Richtertätigkeit an den obersten Bundesgerichten wissenschaftlich geprägt ist, muss eine Bewerberin diese Fähigkeit zu wissenschaftlicher Arbeit nachweisen können, zum Beispiel durch Veröffentlichungen, Promotion, wissenschaftliche Mitarbeit in einem Senat. Letzteres ist besonders zu empfehlen, wenn das Amt einer Bundesrichterin angestrebt wird. Nähere Informationen finden Sie auf der Homepage des djb unter Karriere/ Arbeit/Abordnung und wissenschaftliche Mitarbeit. Sprechen Sie mit Ihrer zuständigen Personalstelle, wenn Sie Interesse an einer Abordnung und später an einer Bundesrichterinnenstelle haben. Lassen Sie sich vor allem nicht vertrösten! Verlangen Sie, wenn sich auf Ihre Interessensbekundung nichts tut, eine klare Antwort, ob Sie in Frage kommen und, wenn ja, wann.
Neben der Suche nach geeigneten Kolleginnen habe ich Anfang November 2011 die Mitglieder des Richterwahlausschusses angeschrieben. 11 Landesjustizministerien haben geantwortet; mit sieben der 16 Bundestagsabgeordnet/inn/en, die dem Wahlausschuss angehören, kam ein Kontakt zustande. Eine besondere Stellung unter den Abgeordneten haben diejenigen, die die Richterwahlen koordinieren. Die sog. Obleute waren unserem Anliegen gegenüber sehr aufgeschlossen. Dennoch hat sich bei der Wahl wieder der Parteien- und Länderproporz zum Nachteil der vorgeschlagenen Frauen durchgesetzt. Zu hoffen ist, dass die inzwischen breite öffentliche Diskussion über das Thema Frauen in Führungspositionen, die der djb mit seinem Projekt „Aktionärinnen fordern Gleichberechtigung “ maßgeblich vorangetrieben hat, nicht nachlässt. Denn offensichtlich muss sich der Druck auf die Politik, in den von ihr selbst verantworteten Bereichen den Frauenanteil zu erhöhen, noch verstärken.

In den Schreiben an die Mitglieder des Richterwahlausschusses habe ich unter anderem danach gefragt, welche Maßnahmen sie für erforderlich halten, um die Zahl der Bundesrichterinnen in absehbarer Zeit signifikant zu erhöhen. Drei Landesjustizminister/innen setzen auf eine konsequente gezielte Förderung von Richterinnen, um einen Pool hoch qualifizierter weiblicher Führungskräfte zu schaffen. Ein solcher Pool ist gerade als Datenbank für weibliche Nachwuchskräfte, die für Aufsichtsratspositionen in Frage kommen, eingerichtet worden. In Brandenburg gibt es bereits ein Programm zur Entwicklung von Führungskräften in der Justiz, an dem überdurchschnittlich viele junge Richterinnen teilnehmen. Vier Justizminister/innen sehen auch die obersten Bundesgerichte in der Pflicht, für eine bessere Vereinbarkeit von Familie und Karriere im richterlichen Dienst zu sorgen, zum Beispiel durch Teilzeitstellen für Bundesrichterinnen und wissenschaftliche Mitarbeiterinnen. Diese Entwicklung gilt es voranzutreiben. Der djb wird sich dafür einsetzen, dass die Karrierepfade vielfältiger und auch für Richterinnen und Staatsanwältinnen mit Kindern gangbarer werden.

Für die Richterwahl 2013 möchte ich versuchen, die Justizminister/innen und die Gerichtspräsident/inn/en der 2. Instanz davon zu überzeugen, die Landesvorschlagslisten für die Richterwahlen in einem transparenten Verfahren, zum Beispiel durch Ausschreibung, zu erstellen. Da das Abgeben von Macht freilich ein langwieriger Prozess ist, werden wir wohl eher mit der Forderung, bei der nächsten Wahl mindestens 40 Prozent Richterinnen vorzuschlagen, Erfolg haben.

\title{
Recht und Gender, Portal der FernUniversität in Hagen
}

\section{Ulrike Schultz \\ Akademische Oberrätin, FernUniversität in Hagen}

„Recht und Gender“ ist ein Portal auf der Plattform der FernUniversität für eine Serie von Video-Interviews zu Themen aus dem Bereich Frauenrecht, Geschlechterfragen im
Recht, Gleichberechtigung, Gleichstellung. Das Portal wird ab Juni 2012 erreichbar sein über: www.fernuni-hagen.de/ RechtundGender.

Die Interviews zu „Recht und Gender“ ergänzen das Studienmaterial des Moduls „Recht der Gleichstellung und Genderkompetenz" im Master of Laws der FernUniversität. 
Dieses Modul wird seit 2008 vom Lehrstuhl für Deutsches und Europäisches Verfassungs- und Verwaltungsrecht sowie Europarecht (Prof. Dr. Andreas Haratsch) angeboten. Es basiert auf Studienmaterialien aus dem früheren weiterbildenden Studium „VINGS (Virtual International Gender Studies)Qualifizierung für Gleichstellungsarbeit“, die auf die neue Zielgruppe fokussiert, außerdem aktualisiert und ergänzt worden sind. Das Gendermodul erfreut sich ständig wachsenden Zuspruchs. Es kann im Rahmen der Akademiestudien der FernUniversität auch unabhängig von einer Einschreibung für den Studiengang als Einzelmodul belegt und studiert werden. Von dieser Möglichkeit machen - wie früher bei VINGSQualifizieren - viele Gleichstellungsbeauftragte Gebrauch. Das Gendermodul hat den Schwerpunkt auf schriftlichem Material, das online über die Plattform „moodle“ angeboten wird.

Für die Idee von „Recht und Gender“ hat das Projekt „Gesellschaft begreifen“ von Prof. Dr. Uwe Schimank und Dr. Nadine Schöneck Pate gestanden. Begleitend zu einem Sammelband mit diesem Titel haben sie im Studienportal des B.A. Soziologie 13 Videoclips mit den Autorinnen und Autoren des Buches eingestellt. ${ }^{1}$

An der FernUniversität in Hagen sind von 1985 bis 1995 Filme zum weiterbildenden Studium „Frauen im Recht“ erstellt worden. Dabei sind Vorträge einer gleichnamigen Vortragsreihe für die damalige Fernsehserie der FernUniversität „FernUniversität im Dritten“ aufgenommen und bearbeitet worden. ${ }^{2}$ An diese Tradition sollte mit den Interviews „Recht und Gender“ angeknüpft werden. Das Ministerium für Innovation, Wissenschaft und Forschung des Landes NRW hat die Produktion finanziell unterstützt.

Schwerpunktmäßig sind Autorinnen und Autoren des Gendermoduls einbezogen worden. Folgende Interviews sind aufgezeichnet worden:

\section{Grundlagen}

- Prof. Dr. Andreas Haratsch, FernUniversität in Hagen: Art. 3 Abs. 2 - Auslegung und Anwendung

- Renate Augstein, BMFSFJ, Berlin: Von der Frauenbewegung zur professionellen Frauenpolitik

- Prof. Dr. Sibylla Flügge, Fachhochschule Frankfurt a.M.: Feministische Rechtswissenschaft

- Dr. Friederike Wapler, Universität Göttingen: Autonomie der Frau in der Rechtsphilosophie

\section{Antidiskriminerung}

- Prof. Dr. Ulrich Battis, Berlin: Verfassungsrechtliche Fragen gleichgeschlechtlichen Zusammenlebens

- Bernhard Franke, Antidiskriminierungsstelle des Bundes, Berlin: Die Antidiskriminierungsstelle und das AGG

\section{Geschlechterfragen im Familien-, Arbeits- Steuer- und Ren- tenrecht}

- Prof. Dr. Sabine Berghahn, Berlin: Das Ernährermodell im deutschen Recht
- Prof. Dr. Margarete Schuler-Harms, Universität der Bundeswehr, Hamburg: Geschlechtergerechte Rentenreform

- Prof. Dr. Ute Sacksofsky, Universität Frankfurt: Einfluss des Steuerrechts auf die Familienpolitik - neue Fragen und Entwicklungen

\section{Staatlicher Schutz vor Gewalt}

- Dr. Birgit Schweikert, BMFSFJ, Berlin: Schutz von Frauen vor Gewalt

- Prof. Dr. Konstanze Plett, Universität Bremen: Intersexualität und Transgender

- Maria Wersig, Universität Hildesheim: Reproduktion zwischen „Lebensschutz“, Selbstbestimmung und Technologie

- Prof. Dr. Susanne Karstedt, Universität Leeds: Ist Strafrecht Männerrecht, oder sind Frauen die tugendhafteren Menschen? Genderfragen in der Kriminologie

\section{Europarecht und Internationales Recht}

- Prof. Dr. Uta Klein, Universität Kiel: Aktuelle Genderfragen im Europarecht

- Prof. Dr. Beate Rudolf, Deutsches Institut für Menschenrechte, Berlin: Das Deutsche Institut für Menschenrechte und die Frauenrechte

- Prof. Dr. Ulrike Lembke, Universität Hamburg: Migration, Flucht und Geschlecht

- Dr. Fatima Kastner, Institut für Sozialforschung Hamburg, Auswirkungen transnationaler Frauenrechtsbewegungen auf lokale Rechtsordnungen

\section{Juristinnen}

- VR'in OLG Brandenburg Ramona Pisal, Präsidentin des Deutschen Juristinnenbundes: Der deutsche Juristinnenbund: Lobby für die Frauen - Netzwerk der Juristinnen

- AOR'Ulrike Schultz, FernUniversität in Hagen: Juristinnen

- Prof. Dr. Ulla Gläßer, Universität Frankfurt a.d. Oder: Genderfragen in der Mediation

Zusätzlich werden Videostreams von Vortragsveranstaltungen in das Portal „Recht und Gender“ eingestellt. Verfügbar sind bisher:

- Ulrike Schultz, FernUniversität in Hagen, 2012: Von der Geschlechtervormundschaft zur Gleichstellung. http://www.fernuni-hagen.de/videostreaming/rewi/ ls_haratsch/20120423.shtml

- Ulrike Schultz, FernUniversität in Hagen, 2011: Europa so fern und doch so nah? Was Frauen über den Einfluss von Europa auf ihr Leben und ihre Rechte wissen sollten. http://www.fernuni-hagen.de/videostreaming/rewi/ ls_haratsch/20110509.shtml

1 〈www.fernuni-hagen.de/ksw/basoz/gesellschaftbegreifen/〉(Zugriff: 23.4.2012).

2 Einige der Videos „Frauen im Recht“ sind im Videoarchiv der FernUni öffentlich zugänglich (http://www.fernuni-hagen.de/ videostreaming/zmi/video/\#rewi). Weitere Filme werden noch eingestellt. Im Internet zugänglich sind auch Videostreams der 2004/2005 durchgeführten Videokonferenzen zu „Equal Opportunities in Comparative Perspective“ (http://www.fernuni-hagen.de/ videostreaming/bwp/) 
- Prof. Dr. Ute Sacksofsky, Universität Frankfurt, 2002: Steuerung der Familie durch Steuern. http://www.fernuni-hagen.de/videostreaming/zmi/video/2002/02-15_00000/

- Prof. Dr. Monika Frommel, Universität Kiel, und Prof. Dr. Jan Beckmann, FernUniversität in Hagen, 2012: Rechtliche und ethische Fragen der Reproduktionsmedizin. http://www. fernuni-hagen.de/videostreaming/gleichstellung/201205/

Geplant u.a.

- Ulrike Spangenberg, Freie Universität Berlin: Geschlechtergerechtigkeit im Steuerrecht?

Die Interviews im Portal „Recht und Gender“ sind frei zugänglich. Damit soll für das Gendermodul geworben und Studierenden der anderen Fakultäten und Studiengänge sowie externen Interessierten die Möglichkeit zur Bearbeitung der Inhalte und damit Stärkung ihrer Genderkompetenz im Recht geboten werden.
Über das Internetportal „Recht und Gender“ werden auch Video-Interviews mit Portraits herausragender Rechtswissenschaftlerinnen zugänglich gemacht. Das erste war mit Richterin am Bundesverfassungsgericht Prof. Dr. Susanne Baer. Die Porträts werden im Kontext des Projekts JurPro aufgezeichnet. JurPro - De jure und de facto: Professorinnen in der Rechtswissenschaft - ist ein vom BMBF über die Förderlinie Frauen an die Spitze gefördertes Drittelmittelprojekt. Es sollen die Bedingungen von Professorinnenkarrieren untersucht und Vorschläge zur Verbesserung der Organisationsstruktur und -kultur in den Rechtswissenschaften erarbeitet werden. vgl. www.fernuni-hagen.de/jurpro

\section{Ansprechpartnerin}

AOR'Ulrike Schultz

Tel.: 02331-870811

Ulrike.Schultz@FernUni-Hagen.de

\section{Rezension: Festschrift 150 Jahre djt}

\author{
150 Jahre Deutscher Juristentag \\ Festschrift Deutscher Juristentag 1860-2010 \\ Festschriften \\ 2010. Buch. XVIII, 763 S. In Leinen \\ C.H.BECK ISBN 978-3-406-59824-1 \\ München 2010 \\ 168,oo Euro inkl. MwSt.
}

Die Festschrift zum 150-jährigen Jubiläum des Deutschen Juristentages (djt) zeichnet ein sowohl sehr umfassendes Bild der inhaltlichen Arbeit und des Einflusses des djt als auch der Vereinsgeschichte und des Vereinslebens. Das umfangreiche Werk beginnt mit einer selbstkritischen Auseinandersetzung mit der Geschichte des Verbandes. Insbesondere wird die stillschweigende Akzeptanz nach 1945 von Juristen in den eigenen Reihen, denen eine nationalsozialistische Gesinnung nachgewiesen werden konnte, beleuchtet. Im Anschluss wird auf über 400 Seiten zum Teil sehr ausführlich für jedes Rechtsgebiet die inhaltliche Arbeit des Juristentages dargestellt. Der dritte Teil der Festschrift befasst sich mit der Außensicht, während sich der vierte und letzte Teil mit der Binnensicht auf den djt beschäftigt.

Für aktive Vereinsmitglieder ist diese Festschrift sicherlich empfehlenswert, für alle anderen ist sie außerordentlich detailliert. Aus Genderperspektive ist zu bedauern, dass trotz zahlreicher ak- tiver Juristinnen im djt nur zwei der 30 Autor/inn/en weiblich sind. Gleichberechtigung und Gleichstellung kommen als Themen fast nur punktuell im Zusammenhang mit Beschlüssen des djt vor. Die frauen- bzw. geschlechterrelevanten Beschlüsse des djt sind jedoch aus frauenrechtspolitischer Sicht nicht immer begrüßenswert. Um nur zwei Beispiele zu nennen: Der djt sprach sich im Jahr 1974 gegen ein Verbot der Frage nach einer Schwangerschaft im Vorstellungsgespräch aus. 30 Jahre später plädierte der djt für eine Abschaffung des Anspruchs auf Teilzeit im Teilzeit- und Befristungsgesetz.

Ein frauenrelevantes Thema, das an verschiedenen Stellen des Buches diskutiert wird, sind die Diskussionen 2000 und 2002 zur Fassung der Satzung in geschlechtergerechter Sprache. Die geschlechtsneutrale Satzung wurde maßgeblich von der damaligen 1 . Vorsitzenden des djb, Ursula Nelles, und weiteren djb-Kolleginnen gefordert. Reinhard Böttcher, Präsident des djt 2000 und 2002, erwähnt in seinem Beitrag, was für eine peinliche Berichterstattung er gefürchtet hatte, wenn die Abstimmung über die neue Fassung der Satzung in geschlechtergerechter Sprache 2002 gescheitert wäre.

Es bleibt für die Zukunft zu hoffen, dass der djt es bei zukünftigen Veröffentlichungen auch als Blamage ansehen wird, für ein solches Werk weniger als zehn Prozent Autorinnen vorweisen zu können. (Elif Beden) 